\title{
Geometric Asian Option Pricing in General Affine Stochastic Volatility Models with Jumps
}

\author{
Friedrich Hubalek, \\ Vienna University of Technology, Financial and Actuarial Mathematics, \\ Wiedner Hauptstraße 8/105-1, A-1040 Vienna, Austria (fhubalek@fam.tuwien.ac.at) \\ Martin Keller-Ressel, \\ Institut für Mathematik, TU Berlin, \\ Strasse des 17. Juni 136, D-10623 Berlin, Germany (mkeller@math.tu-berlin.de) \\ Carlo Sgarra* \\ Department of Mathematics, Politecnico di Milano, \\ Piazza Leonardo da Vinci, 32, I-20133 Milan, Italy (carlo.sgarra@polimi.it)
}

July 10, 2014

\begin{abstract}
In this paper we present some results on Geometric Asian option valuation for affine stochastic volatility models with jumps. We shall provide a general framework into which several different valuation problems based on some average process can be cast, and we shall obtain close-form solutions for some relevant affine model classes.

Keywords: Geometric Asian Options, Average Strike Options, Average Price Options, Stochastic Volatility, Affine Processes.
\end{abstract}

\section{Introduction}

Asian options are quite common derivatives often combined with other financial claims in order to construct structured products [Kat01; they can in fact provide protection against strong price fluctuations in volatile markets and reduce the possibilities of market manipulations near the expiry. That is because Asian options are roughly speaking options on the average value assumed by the underlying during the option's life, and they require some mathematical effort in order to describe the dynamics of the average under consideration. For these reasons it is interesting to develop realistic financial models and efficient numerical algorithms to evaluate these kind of options.

Asian options are usually grouped into two main classes according to their payoff: if $S_{T}$ is the value of the underlying asset at maturity $T, K$ is the strike price, and $A_{T}$ is a suitably defined average of the values assumed by the stock during the period under consideration, the so called "Average Strike" (sometimes called "Floating Strike") Asian Calls have the payoff given by the following expression: $\left(S_{T}-A_{T}\right)_{+}$, while the payoff of the "Average Price" (Sometimes called "Fixed Strike" or "Average Rate") Asian Calls is given by: $\left(A_{T}-K\right)_{+}$. The average considered

\footnotetext{
${ }^{*}$ Corresponding author. Tel.: +3902 2399 4570; fax: +39 0223994621
} 
can be the Geometric or the Arithmetic one and it can be calculated on a continuous or discrete monitoring basis. All these details are specified by the contracts stipulated by two counterparts, as Asian options are mainly OTC (Over the Counter) traded financial derivatives.

Several results are available on Arithmetic Asian options. In the classical Black-Scholes framework the papers by H.Geman, M. Yor GY93 and by D. Dufresne Duf01 present an evaluation approach based on exponential functionals of Brownian motion properties, while in another paper by D. Dufresne [Duf05], some explicit valuation formulas related to the Bessel process are given. More recently in the paper by M. Schröder [Sch08] a comprehensive analysis of the Arithmetic Asian options is provided, emphasizing the role plaid by complex analysis and special functions in solving the main valuation problems. In a more general Exponential Lévy setting some results on Arithmetic Asian options are included in the papers by H. Albrecher and M. Predota AP04, where the Lévy process describing the underlying evolution is assumed to be of NIG type, and by H. Albrecher Alb04. When explicit formulas do not exist some accurate analytic approximations have been proposed, like in MP98. The paper by J. Večer and M. $\mathrm{Xu}$ [VX04] is the only one, to our knowledge, dealing with the valuation problem of Arithmetic Asian options in a general semimartingale setting, where a Partial Integro-Differential Equation is provided solving the problem in the special case of an underlying described by a process with independent increments. The paper by J.-P. Fouque and C.-H. Han FH03 deals with the evaluation problem for Arithmetic Asian options in a stochastic volatility framework by extending the reduction technique introduced by J. Večer and M. Xu VX04. As far as lower and upper bounds on prices are concerned some results are available for Arithmetic Asian options both in the continuous SGD00, and discrete monitoring case $\mathrm{VDL}^{+} 06$, where a convenient use of the comonotonicity property is exploited in order to provide such bounds. The Hedging issue of Asian options has been considered in AG03, where a static strategy is examined.

As far as Geometric Asian Options are concerned, their evaluation in the basic Black-Scholes setting is very simple. While a direct argument can provide an explicit solution for Geometric Average Rate Calls (under continuous monitoring), a slightly more involved calculation can provide at least an accurate numerical approximation for the Average Strike Call (still under continuous monitoring) in the same framework [WHD95. As far as the Lévy models are concerned, several results are also available: we recall here the paper by C.B. Zhang and C.W. Oosterlee [ZO13]. For the discrete monitoring case some definite results for Lévy models are illustrated in [FM08].

It has been pointed out Gla04 that the Geometric Asian option pricing is extremely useful also for the arithmetic average option valuation via Monte Carlo methods with control variables.

As far as stochastic volatility models are concerned, while Y.L. Cheung and H.Y. Wong CW04b obtain via a perturbation method some semi-analytical formulas for Geometric Asian options in stochastic volatility models exhibiting a mean-reverting behavior. The paper by I. Peng deals with Geometric Asian options valuation in a local volatility setting, namely in the CEV model [Pen06]. More recent results, standing on Asymptotic expansion techniques, have been obtained by E. Gobet and M. Miri GM11. In a very recent paper by B. Kim and I.-S. Wee [KW11] the Geometric Asian option pricing problem has been studied for the stochastic volatility model proposed by Heston.

The intrinsic limitations of the Black-Scholes model are well known since a long time. In particular, the fat tail, the volatility clustering, the aggregational gaussianity features exhibited by stock prices distributions, and moreover the volatility smiles and the leverage effect empirically observed cannot be explained by this model. While Lévy-based and stochastic volatility models can explain some of these phenomena separately, the models including both features, i.e. stochastic volatility and jumps, can provide a much more realistic description of stock prices behavior. Several models of this type have been proposed in the literature and we just mention here the models suggested by D. Bates Bat96], Bat00, by O.E. Barndorff-Nielsen and N. Shephard 
BNS01, BNNS02, and the Time-Changed Lévy models proposed by P. Carr, H. Geman, D. Madan and M. Yor CGMY03, CW04a among others. The price to pay for this substantial improvement in modeling is a bigger difficulty in performing calculations for evaluating derivatives. Very few results are available in this more general setting.

In HS11 a semi-explicit evaluation formula for Geometric Asian Options, for fixed and floating strike, under continuous monitoring, when both stochastic volatility and jumps come into play has been provided; in that paper a specific model framework was considered, i.e. the Barndorff-Nielsen and Shephard model.

Almost all of the above mentioned pricing models in which stochastic volatility features have been combined with jumps belong to the large family of affine models, according to the definition provided by D. Duffie, D. Filipovic and W. Schachermayer DFS03. This class includes almost all the most popular pricing models existing in the literature related to many different type of underlying assets: fixed income securities, credit risk models, equities and commodities. Many relevant features of these models can be described in a unified way by the very general framework provided by the affine process approach. For an extensive treatment of the general properties of affine models and some related technical issues we mention the Thesis by M. Keller-Ressel KR08.

We want to recall here another relevant class of valuation problems requiring the description of some average process, i.e. options on realized volatility and variance swaps. Several recent papers attacked these valuation problems in different setting. In the paper by J. Kallsen, J. Muhle-Karbe and M. Voss [KMKV11 the pricing of options on variance in affine stochastic volatility models has been extensively investigated; some results in a Barndorff-Nielsen and Shephard modeling framework were provided in [BGK07, while in [CLW12] variance swaps pricing has been studied for time-changed Lévy models.

The contribution of the present paper is to develop a general valuation scheme and to provide some semi-explicit evaluation formulas for Geometric Asian Options, when the underlying process describing the joint dynamics of logreturns and volatility is affine. We shall provide a quite general framework into which several different valuation problems can be formulated and solved: all those based on the geometric mean of the variables, including average price and average strike Asian call options; variance options valuation can be also cast into the present framework, but only for continuous return processes, as we shall discuss in Section 3 .

In next section we shall introduce the general setting and the notations used throughout the paper, while in Section 3 we shall present the introductory results on the affine representation for integral functionals. After providing in Section 4 an auxiliary result based on a change-ofnumeraire technique, which will turn out to be useful for Average Strike option calculations, in Section 5 we shall present the general results on Geometric Asian options valuation in a general affine framework. In Section 6] we shall apply our general results to the most popular concrete affine stochastic volatility models and we shall provide the semi-explicit formulas for both Average Price and Average Strike options for these models. In section 7 we shall resume the main results obtained in this paper and we'll outline some possible developments of the present work.

\section{Model Setup}

The purpose of this section is to clarify the framework in which we are going to develop our pricing problem and to clarify the basic notations adopted in the following. The results recalled here are mainly based on the treatment provided in [KR08] and KR11]. We fix some time horizon $T>0$ up to which we wish to model the price process of some financial asset. Let $\left(\Omega, \mathcal{F},\left(\mathcal{F}_{t}\right)_{t \in[0, T]}, \mathbb{P}\right)$ 
be a filtered probability space, which supports all the processes we encounter in the sequel.

We shall call an affine process a stochastically continuous, time-homogeneous Markov process $\left(X_{t}, \mathbb{P}^{x}\right)$ with state space $D=\mathbb{R}_{+}^{m} \times \mathbb{R}^{n}$ if its characteristic function is an exponentially affine function of the state vector, i.e., if there exist functions $\phi: \mathbb{R}_{+} \times \mathcal{U} \rightarrow \mathbb{C}_{-}, \boldsymbol{\psi}: \mathbb{R}_{+} \times \mathcal{U} \rightarrow \mathcal{U}$ such that

$$
\log \left(\mathbb{E}\left[\exp \boldsymbol{u} \cdot \boldsymbol{X}_{t} \mid \boldsymbol{X}_{0}\right]\right)=\phi(t, \boldsymbol{u})+\boldsymbol{X}_{0} \cdot \boldsymbol{\psi}(t, \boldsymbol{u})
$$

for all $(t, \boldsymbol{u}) \in \mathbb{R}_{+} \times \mathcal{U}$ and where

$$
\mathbb{C}_{-}:=\{u \in \mathbb{C}: \Re u \leq 0\} \text { and } \mathcal{U}:=\mathbb{C}_{-}^{m} \times i \mathbb{R}^{n} .
$$

By convention, the logarithm above denotes the distinguished logarithm in complex plane, that makes $\phi$ and $\psi$ jointly continuous in the complex plane (cf. [DFS03]). Note that due to the Markov property an analogous equation also holds true for expectations conditional on $\boldsymbol{X}_{s}$, that is

$$
\log \left(\mathbb{E}\left[\exp \boldsymbol{u} \cdot \boldsymbol{X}_{t} \mid \boldsymbol{X}_{s}\right]\right)=\phi(t-s, \boldsymbol{u})+\boldsymbol{X}_{s} \cdot \boldsymbol{\psi}(t-s, \boldsymbol{u})
$$

for all $0 \leq s \leq t$ and $\boldsymbol{u} \in \mathcal{U}$. An affine process is called regular if the derivatives:

$$
F(\boldsymbol{u}):=\left.\frac{\partial \phi}{\partial t}(t, \boldsymbol{u})\right|_{t=0^{+}}, \mathbf{R}(\boldsymbol{u}):=\left.\frac{\partial \boldsymbol{\psi}}{\partial t}(t, \boldsymbol{u})\right|_{t=0^{+}},
$$

exist for all $\boldsymbol{u} \in \mathcal{U}$, and are continuous at $\boldsymbol{u}=0$. It has been shown in [KRST10] that any affine process in the sense of the above definition is regular and hence that the functions $F(\boldsymbol{u})$ and $R(\boldsymbol{u})$ are well-defined.

Since the functions $F(u)$ and $\boldsymbol{R}(\boldsymbol{u})$ completely characterize the process $\left(\boldsymbol{X}_{t}\right)_{t>0}$ they are called the functional characteristics of $\left(\boldsymbol{X}_{t}\right)_{t \geq 0}$.

In the following we shall need the notion of truncation function, but we'll specify which truncation function will be used whenever it will be necessary. When an affine process will be assumed to describe the price dynamics of some asset, we shall refer to it as an affine pricing model.

In the following we shall assume the (risk-neutral) stock price process $S_{t}$ to be given as

$$
S_{t}=\exp \left\{(r-q) t+X_{t}\right\}
$$

where $r$ is the risk-free interest rate, $q$ is the dividend yield and $X_{t}$ is the discounted dividendcorrected log-price process.

Let $V_{t}$ denote another (one-dimensional) process with $V_{0}>0$, such that $\left(X_{t}, V_{t}\right)$ is a stochastically continuous, time-homogeneous Markov process.

We define the process $\left(X_{t}, V_{t}\right)$ an Affine Stochastic Volatility model if the cumulant generating function of $\left(X_{t}, V_{t}\right)$ is of the special affine form

$$
\log \left(\mathbb{E}\left[\exp \left\{u X_{t}+w V_{t}\right\} \mid X_{0}, V_{0}\right]\right)=\phi(t, u, w)+V_{0} \psi(t, u, w)+X_{0} u .
$$

Note that this setup is as in [KR11, Section 5], from where we will adopt the nomenclature and call $\left(X_{t}, V_{t}\right)$ affine stochastic volatility $(\mathrm{ASV})$ process and the associated asset price model ASV model.

Remark 1. A bivariate affine model has functional characteristics $F$ and $\boldsymbol{R}=\left(R_{1}, R_{2}\right)$. An $A S V$ has $R_{1}=0$ and we set $R=R_{2}$ and call simply $F, R$ the functional characteristics.

The following theorem characterizes regular ASV processes and provides a representation result for the functions $F, R$. 
Theorem 1. [DFS03, Theorem 2.7] Let $\left(X_{t}, V_{t}\right)_{t>0}$ be a regular ASV process. Then there exist a set of parameters $(a, \alpha, b, \beta, c, \gamma, m, \mu)$ whrer $a, \alpha$ are positive semi-definite matrices, $b, \beta \in \mathbb{R}^{2}$, $c, \gamma \geq 0$ and $m, \mu$ are Lévy measures on $\mathbb{R}^{2}$, such that

$$
\begin{aligned}
& F(u, w)=\frac{1}{2}(u, w) \cdot a \cdot(u, w)^{T}+b \cdot(u, w)^{T}-c+\int_{D \backslash\{0\}}\left(e^{x u+y w}-1-h_{F}(x, y) \cdot(u, w)^{T}\right) m(d x, d y) \\
& R(u, w)=\frac{1}{2}(u, w) \cdot \alpha \cdot(u, w)^{T}+\beta \cdot(u, w)^{T}-\gamma+\int_{D \backslash\{0\}}\left(e^{x u+y w}-1-h_{R}(x, y) \cdot(u, w)^{T}\right) \mu(d x, d y)
\end{aligned}
$$

holds, where $h_{F}(x, y), h_{R}(x, y)$ are suitable truncation functions. Furthermore the functions $\phi$ and $\psi$ in (5) fulfill the generalized Riccati equations:

$$
\begin{aligned}
\partial_{t} \phi(t, u, w) & =F(u, \psi(t, u, w)), & & \phi(0, u, w)=0, \\
\partial_{t} \psi(t, u, w) & =R(u, \psi(t, u, w)), & & \psi(0, u, w)=w .
\end{aligned}
$$

For option pricing we employ a structure preserving martingale measure. This means, we choose an equivalent martingale measure, such that the model structure remains unchanged, only model parameters change. For several particular models enjoying the affine structure, a systematic investigation has been performed on the class of equivalent martingale measure, also providing a full characterization of the subclass of structure preserving measures: for the BNS model we mention the paper by E. Nicolato and E. Venardos [NV03, while for the Bates model a brief discussion on the subject is included in Bat96.

The following proposition provides a sufficient condition for an affine process to be conservative (i.e. non-exploding) and a martingale:

Proposition 1. [KR11, Corollary 2.1] Let $\left(X_{t}, V_{t}\right)$ be defined as before and the quantity $\chi(u)$ be defined as follows:

$$
\chi(u):=\left.\frac{\partial R}{\partial w}(u, w)\right|_{w=0} .
$$

If $F(0,0)=R(0,0)=F(1,0)=R(1,0)=0$ and $\max \{\chi(0), \chi(1)\}<\infty$, then $\exp \left\{X_{t}\right\}$ is a conservative process and a martingale.

Note that $F(0,0)=R(0,0)=0$ is equivalent to $c=\gamma=0$.

\section{Integral functionals for ASV models}

Our starting point is an affine ASV model $(X, V)$ as introduced above. To study Geometric Asian options or realized variance options we introduce the associated integral processes $Y$ and $Z$ with

$$
Y_{t}=\int_{0}^{t} X_{s} d s, \quad Z_{t}=\int_{0}^{t} V_{s} d s .
$$

Proposition 2. If $(X, V)$ is an $A S V$ model with functional characteristics $(F, R)$, then the joint law of $\left(X_{t}, V_{t}, Y_{t}, Z_{t}\right)$ is described by

$$
\log E\left[e^{u_{1} X_{t}+u_{2} V_{t}+u_{3} Y_{t}+u_{4} Z_{t}} \mid X_{0}, V_{0}\right]=\Phi\left(t, u_{1}, u_{2}, u_{3}, u_{4}\right)+\left(u_{1}+u_{3} t\right) X_{0}+\Psi\left(t, u_{1}, u_{2}, u_{3}, u_{4}\right) V_{0}
$$

where

$$
\begin{array}{ll}
\dot{\Phi}=F\left(u_{1}+u_{3} t, \Psi\right) & \Phi(0)=0 \\
\dot{\Psi}=R\left(u_{1}+u_{3} t, \Psi\right)+u_{4} & \Psi(0)=u_{2} .
\end{array}
$$


Proof. It follows from [KR08, Theorem 4.10, p.50] for two dimensions, that $(X, V, Y, Z)$ is affine,

$$
\log E\left[e^{u_{1} X_{t}+u_{2} V_{t}+u_{3} Y_{t}+u_{4} Z_{t}} \mid X_{0}, V_{0}, Y_{0}, Z_{0}\right]=\Phi(t)+\psi_{1}(t) X_{0}+\psi_{2}(t) V_{0}+\psi_{3}(t) Y_{0}+\psi_{4}(t) Z_{0},
$$

where the $\Phi$ and $\psi_{i}$ satisfy the Riccati equations

$$
\begin{array}{ll}
\dot{\Phi}=F\left(\psi_{1}, \psi_{2}\right) & \Phi(0)=0 \\
\dot{\psi}_{1}=\psi_{3} & \psi_{1}(0)=u_{1} \\
\dot{\psi}_{2}=R\left(\psi_{1}, \psi_{2}\right)+\psi_{4} & \psi_{2}(0)=u_{2} \\
\dot{\psi}_{3}=0 & \psi_{3}(0)=u_{3} \\
\dot{\psi}_{4}=0 & \psi_{4}(0)=u_{4} .
\end{array}
$$

Remember that the solutions of (13) depend on the parameters $u_{1}, u_{2}, u_{3}, u_{4}$, thus $\psi_{1}(t)=$ $\psi_{1}\left(t ; u_{1}, u_{2}, u_{3}, u_{4}\right)$ etc. Some of those equations can be immediately integrated. Obviously $\psi_{3}(t)=u_{3}, \psi_{4}(t)=u_{4}, \psi_{1}(t)=u_{1}+u_{3} t$ and the only relevant equations are

$$
\begin{array}{ll}
\dot{\Phi}=F\left(u_{1}+u_{3} t, \psi_{2}\right) & \Phi(0)=0 \\
\dot{\psi}_{2}=R\left(u_{1}+u_{3} t, \psi_{2}\right)+u_{4} & \psi_{2}(0)=u_{2}
\end{array}
$$

Then we note that $Y_{0}=0$ and $Z_{0}=0$ and we set $\Psi=\psi_{2}$. (36) and (37) follow from (18) and (19).

Remark 2. Variance swaps and options on realized variance in stochastic volatility models with jumps have been studied in [BGK07] and [Sep08]. In a general affince setting they have been investigated extensively in the paper [KMKV11] where the realized variance is approximated by the quadratic variation of the log-return process. For continuous return processes, such as the Heston model, for example, the quadratic variation $[X, X]$ and its predictable part $\langle X, X\rangle$ coincide with integrated variance, which is our $Z$.

The cumulant of the integrated variance can be computed according to the following corollary, which turns out to be a special case of [KMKV11, Lemma 5.1, P.634].

\section{Corollary 1.}

$$
\log E\left[e^{w Z_{t}}\right]=\phi(t, w)+V_{0} \psi(t, w)
$$

where

$$
\begin{array}{ll}
\dot{\phi}=F(0, \psi) & \phi(0)=0 \\
\dot{\psi}=R(0, \psi)+w & \psi(0)=0
\end{array}
$$

Proof. This follows immediately from Prop 2 with $u_{1}=0, u_{2}=0, u_{3}=0, u_{4}=w$.

\section{Change of numeraire for ASV models}

To calculate the price of the average strike option we apply the change-of-numeraire technique and take the stock as a new numeraire.

From now on we denote the martingale measures with the bond resp. stock as a numeraire by $Q^{0}$ resp. $Q^{1}$, and expectations $E^{0}$ resp. $E^{1}$.

$$
\log E_{x, v}^{0}\left[e^{u_{1} X(t)+u_{2} V(t)}\right]=\phi^{0}\left(t, u_{1}, u_{2}\right)+x \psi_{1}^{0}\left(t, u_{1}, u_{2}\right)+v \psi_{2}^{0}\left(t, u_{1}, u_{2}\right)
$$


Thus we have the density process

$$
\frac{d Q^{1}}{d Q^{0}}(t)=e^{X_{t}-x}
$$

on $\mathcal{F}_{t}$.

Let's start with the following

Lemma 1. If $(X, V)$ is affine under $Q^{0}$ with functional characteristics $F^{0}$ and $R^{0}$, then it is affine under $Q^{1}$ with functional characteristics $F^{1}$ and $R^{1}$ given by

$$
F^{1}\left(u_{1}, u_{2}\right)=F^{0}\left(u_{1}+1, u_{2}\right), \quad R^{1}\left(u_{1}, u_{2}\right)=R^{0}\left(u_{1}+1, u_{2}\right)
$$

Proof.

$$
\begin{gathered}
\log E_{x, v}^{1}\left[e^{u_{1} X(t)+u_{2} V(t)}\right]=\log E_{x, v}^{0}\left[e^{x+X_{t}} \cdot e^{u_{1} X(t)+u_{2} V(t)}\right]= \\
-x+\log E_{x, v}^{0}\left[e^{\left(u_{1}+1\right) X(t)+u_{2} V(t)}\right]= \\
\phi^{0}\left(t, u_{1}+1, u_{2}\right)+x\left(\psi_{1}^{0}\left(t, u_{1}+1, u_{2}\right)-1\right)+v \psi_{2}^{0}\left(t, u_{1}+1, u_{2}\right)= \\
\phi^{1}\left(t, u_{1}, u_{2}\right)+x \psi_{1}^{1}\left(t, u_{1}, u_{2}\right)+v \psi_{2}^{1}\left(t, u_{1}, u_{2}\right)
\end{gathered}
$$

with

$$
\begin{aligned}
& \phi^{1}\left(t, u_{1}, u_{2}\right)=\phi^{0}\left(t, u_{1}+1, u_{2}\right), \\
& \psi_{1}^{1}\left(t, u_{1}, u_{2}\right)=\psi_{1}^{0}\left(t, u_{1}+1, u_{2}\right)-1, \\
& \psi_{2}^{1}\left(t, u_{1}, u_{2}\right)=\psi_{2}^{0}\left(t, u_{1}+1, u_{2}\right)
\end{aligned}
$$

Thus

$$
F^{1}\left(u_{1}, u_{2}\right)=F^{0}\left(u_{1}+1, u_{2}\right) \quad R^{1}\left(u_{1}, u_{2}\right)=R^{0}\left(u_{1}+1, u_{2}\right),
$$

If $e^{X}$ is a martingale we have $F^{0}(1,0)=R^{0}(1,0)=0$ and thus $F^{1}(0,0)=R^{1}(0,0)=0$.

Lemma 2. If $(X, V)$ is an $A S V$ model, then the joint law of $\left(X_{t}, Y_{t}\right)$ under $Q^{1}$ is described by

$$
\log E^{1}\left[e^{u X_{t}+w Y_{t}}\right]=\phi^{1}(t, u, w)+v \psi^{1}(t, u, w)
$$

where

$$
\begin{array}{ll}
\left(\phi^{1}\right)^{\prime}=F\left(u+1, \psi^{1}\right) & \phi^{1}(0)=0 \\
\left(\psi^{1}\right)^{\prime}=R\left(u+1, \psi^{1}\right) & \psi^{1}(0)=w .
\end{array}
$$

Proof. This follows from Lemma 1 and Proposition 2 applied to $Q^{1}$ resp. $F^{1}, R^{1}$.

\section{General results for Geometric Asian options}

\subsection{Average price}

Let us denote by $\bar{X}_{T}$ the arithmetic average of the log-returns process and by $\hat{S}_{T}$ the geometric average of the stock prices, then

$$
\bar{X}_{T}=(r-q)+\frac{1}{T} \int_{0}^{T} X_{s} d s, \quad \hat{S}_{T}=e^{\bar{X}_{T}}=\exp \left((r-q)+\frac{1}{T} \int_{0}^{T} X_{s} d s\right) .
$$

For average strike we shall need the cumulant of integrated log-returns. 
Corollary 2. If $(X, V)$ is an $A S V$ model, then the law of $Y_{t}=\int_{0}^{t} X_{s} d s$ is described by

$$
\log E\left[e^{w Y_{t}}\right]=\Phi(t, w)+w t X_{0}+V_{0} \psi(t, w)
$$

where

$$
\begin{aligned}
\dot{\Phi} & =F(w t, \psi) & \Phi(0) & =0 \\
\dot{\psi} & =R(w t, \psi) & \psi(0) & =0 .
\end{aligned}
$$

Proof. This follows immediately from Prop 2 with $u_{1}=0, u_{2}=0, u_{3}=w, u_{4}=0$.

Theorem 2. Assume there exists $a>1$ such that

$$
E\left[e^{a \bar{X}_{T}}\right]<\infty,
$$

then the time-zero value of an average price Asian call option is given by

$$
E\left[e^{-r T}\left(\hat{S}_{T}-K\right)_{+}\right]=\frac{e^{-r T}}{2 \pi i} \int_{a-i \infty}^{a+i \infty}\left(\frac{1}{K}\right)^{u} \frac{K}{u(u-1)} e^{\kappa(T, u)} d u,
$$

with the cumulant function $\kappa(T, u)=\log E\left[e^{u \bar{X}_{T}}\right]$. It is given by

$$
\kappa(T, u)=u(r-q)+\phi(T, u)+u X_{0}+\psi(T, u) V_{0},
$$

where

$$
\begin{array}{ll}
\dot{\phi}=F\left(\frac{u t}{T}, \psi\right) & \phi(0)=0 \\
\dot{\psi}=R\left(\frac{u t}{T}, \psi\right) & \psi(0)=0 .
\end{array}
$$

Proof. In order to evaluate the expectation (39) we first use the integral representation (132), which yields

$$
\left(\hat{S}_{T}-K\right)_{+}=\frac{1}{2 \pi i} \int_{a-i \infty}^{a+i \infty}\left(\frac{1}{K}\right)^{u} \frac{K}{u(u-1)} e^{u \bar{X}_{t}} d u,
$$

and then apply Fubini's Theorem, see also [HKK06, and HS11.

Remark 3. The integrability condition (38) guarantees the existence of the cumulant function $\kappa(T, u)$ at $\Re u=a$. It will imply some restrictions on the parameters of the concrete models studied in Section [6. By the results in [?] it is equivalent to the existence to solutions of the Riccati equations (41) for the parameter value $u=a$. The proper set of parameters can be determined individually for each concrete model by studying the real singularities of the cumulant functions, see Doe71, Satz 3.4.1, P.153f], though we are not going to give all details for all models in the example section below. 


\subsection{Average strike}

Theorem 3. If there exists $b<0$ such that

$$
E\left[e^{b \bar{X}_{T}}\right]<\infty
$$

then the time-zero value of an average strike Asian call option is given by

$$
E\left[e^{-r T}\left(S_{T}-\hat{S}_{T}\right)_{+}\right]=\frac{e^{-q T}}{2 \pi i} \int_{b-i \infty}^{b+i \infty} \frac{1}{u(u-1)} e^{\kappa(T, u)} d u
$$

where $\kappa(T, u)=\log E\left[e^{u \bar{X}_{T}+(1-u) X_{T}}\right]$. It is given by

$$
\kappa(T, u)=u(r-q)+\phi(T, u)+V_{0} \psi(T, u)+X_{0}
$$

where

$$
\begin{array}{ll}
\dot{\phi}=F\left(\frac{u t}{T}+(1-u), \psi\right) & \phi(0)=0 \\
\dot{\psi}=R\left(\frac{u t}{T}+(1-u), \psi\right) & \psi(0)=0 .
\end{array}
$$

Proof. Using the change-of-numeraire technique with the density process (24) we obtain

$$
E^{0}\left[\left(S_{T}-\hat{S}_{T}\right)_{+}\right]=e^{(r-q) T} E^{1}\left[\left(1-e^{\bar{X}_{T}-X_{T}}\right)_{+}\right] .
$$

This is just the payoff of a put option on $e^{\bar{X}_{T}-X_{T}}$ with asset and strike both equal to 1 .

The function $\kappa$ is the cumulant function of $\bar{X}_{T}-X_{T}$, which can be obtained from the joint cumulant of $Y_{T}$ and $X_{T}$ in terms of the functions $\phi$ and $\psi$ from Lemma 2.

Similar to the proof of Theorem 2, we can now apply the Laplace integral formula (133) provided in the appendix and Fubini's Theorem to obtain the result.

Remark 4. For the integrability condition (44) a remark similar to Remark 3 above applies.

Proposition 3. Average strike and price Riccati equations have the same structure provided the parameters are changed in the following way. $u \mapsto u+\frac{t}{T}(1-u)$.

Remark 5. The property just described in the proposition above is actually a particular case of a general result called the duality principles in option pricing. This basic property has been systematically investigated in a general semimartingale setting in [Pap07] and in [EPS08].

\section{Geometric Asian options for concrete affine stochastic volatility models}

We now discuss some popular ASV models from the finance literature (for a very nice summary and many more examples, the interested reader is refered to [Kal06]). For a few relevant cases we will obtain an explicit solution of the corresponding Riccati equations. Let us recall, that for all models the asset price will be modeled by $S_{t}=e^{(r-q) t+X_{t}}$, where $X$ denotes the discounted $\log$-price.

In the following examples we shall continue to assume that the model parameters will verify the conditions in Proposition 1, and consequently $e^{X_{t}}$ is a martingale. 


\subsection{Heston model}

The Heston Hes93 model describes the volatility dynamics by means of a CIR-type stochastic differential equation with mean reversion.

The evolution of the discounted log-returns under the risk-neutral measure is then given by

$$
\begin{aligned}
& d X_{t}=\left(-\frac{1}{2} V_{t}\right) d t+\sqrt{V_{t}} d W_{t}^{1}, \\
& d V_{t}=\lambda\left(\theta-V_{t}\right) d t+\zeta \sqrt{V_{t}} d W_{t}^{2},
\end{aligned}
$$

where $\lambda, \theta$, and $\zeta$ are strictly positive parameters. Moreover, in (50) and (51) $W^{1}$ and $W^{2}$ are standard Wiener processes having constant correlation $\rho \in[-1,+1]$.

It can be shown that, if the following condition is satisfied:

$$
\zeta^{2}<2 \lambda \theta
$$

then the volatility process $V$ remains strictly positive (see [Fel51]).

The affine characteristics are KR08, KR11

$$
F(u, w)=\lambda \theta w, \quad R(u, w)=\frac{1}{2}\left(u^{2}-u\right)+\frac{\zeta^{2}}{2} w^{2}-\lambda w+u w \rho \zeta .
$$

\section{Average price}

Combining (53) and (41) we obtain the Riccati equation for the average price

$$
\begin{aligned}
& \dot{\phi}=\lambda \theta \psi, \quad \phi(0)=0 \\
& \dot{\psi}=\frac{\zeta^{2}}{2} \psi^{2}-(\lambda-\rho \zeta u t / T) \psi+\frac{1}{2} u t / T(u t / T-1), \quad \psi(0)=0
\end{aligned}
$$

By using a standard substitution

$$
\psi(t)=\frac{2}{\zeta^{2}} \frac{y^{\prime}(t)}{y(t)}
$$

the Riccati equation can be transformed into a linear differential equation of second order Rei72, PZ03

$$
y^{\prime \prime}+(\lambda-\rho \zeta u t / T) y^{\prime}+\frac{\zeta^{2}}{4} u t / T(u t / T-1)=0 .
$$

The general solution of this equation can be written as a linear combination of two confluent hypergeometric functions of the first kind Sla60] (most commonly denoted by ${ }_{1} F_{1}(a, b, c)$ )

$$
\begin{gathered}
y(t)=C_{1} y_{1}(t)+C_{2} y_{2}(t) \\
y_{1}(t)=A M\left(a_{1}, \frac{1}{2}, c\right) \\
y_{2}(t)=A B M\left(a_{1}+\frac{1}{2}, \frac{3}{2}, c\right)
\end{gathered}
$$

where $a 1, c$ and $A, B$ are defined by the following exressions:

$$
a_{1}=\frac{1}{8} \frac{-\zeta^{2}-2 \rho \zeta(\xi u / T-\lambda)-2 \lambda^{2}}{\zeta u / T \xi^{\frac{3}{2}}}+\frac{1}{4}
$$




$$
\begin{gathered}
c=\frac{1}{2} \frac{((1+t u / T \zeta) \xi-\lambda \rho)^{2}}{\zeta u / T \xi^{\frac{3}{2}}} \\
\xi=\rho^{2}-2 \\
A=\exp -\frac{1}{4}\left[t\left(2 \lambda-\rho \zeta u t / T+\frac{\rho^{2} \zeta u t / T-2 \lambda \rho+2 \zeta(1-u t / T)}{\xi^{\frac{1}{2}}}\right]\right. \\
B=\xi u t / T \zeta-\xi+\lambda \rho
\end{gathered}
$$

By taking into account the initial condition $\psi(0)=0$ we obtain

$$
\psi(t)=-\frac{2}{\zeta^{2}} \frac{y_{2}^{\prime}(0) y_{1}^{\prime}(t)-y_{1}^{\prime}(0) y_{2}^{\prime}(t)}{y_{2}^{\prime}(0) y_{1}(t)-y_{1}^{\prime}(0) y_{2}(t)} .
$$

In view of the (56) we express also $\phi$ explicitly by hypergeometric functions, namely

$$
\phi(t)=-\lambda \theta \frac{2}{\zeta^{2}} \ln \frac{y_{2}^{\prime}(0) y_{1}(t)-y_{1}^{\prime}(0) y_{2}(t)}{y_{2}^{\prime}(0) y_{1}(0)-y_{1}^{\prime}(0) y_{2}(0)}
$$

\section{Average strike}

Combining (53) and (114) with $(u, w) \mapsto(-u, u / T)$ we obtain

$$
\begin{array}{ll}
\dot{\phi}=\lambda \theta \psi+q-r & \phi(0)=0 \\
\dot{\psi}=\frac{1}{2} u^{2}(t / T-1)^{2}+u(t / T-1)+\frac{\zeta^{2}}{2} \psi^{2}-\lambda \psi+\rho \zeta(u(t / T-1)+1) \psi . & \psi(0)=0
\end{array}
$$

The solution to these equations can be obtained in a similar way, providing the expressions for $\phi$ and $\psi$ analogous to (66) and (67) with $y_{1}, y_{2}$ replaced by

$$
\bar{y}_{1}(t)=M\left(\bar{a}_{1}, \frac{1}{2}, \bar{c}\right)
$$

and

$$
\bar{y}_{2}(t)=\bar{A} \bar{B} M\left(\bar{a}_{1}+\frac{1}{2}, \frac{3}{2}, \bar{c}\right) .
$$

where $\bar{a}_{1}, \bar{c}, \bar{A}$ and $\bar{B}$ are now defined by:

$$
\begin{gathered}
\bar{a}_{1}=\frac{1}{8} \frac{\rho \zeta(2 \xi u+\lambda T)-\left(\lambda^{2}+\frac{1}{4} \zeta^{2}\right) T}{\zeta u \xi^{\frac{3}{2}}}+\frac{1}{4} \\
\bar{c}=\frac{1}{2} \frac{\left[\left(\rho^{2}(u-1)+\left(\frac{1}{2}-u\right)\right] T+\zeta u t\left(1-\rho^{2}\right)+\lambda \rho T\right)^{2}}{\zeta u T \xi^{\frac{3}{2}}} \\
\bar{A}=\exp \frac{1}{2}\left[\frac{t}{T}\left(\rho \zeta\left((u-1) T-\frac{1}{2} u t\right)+\lambda T\right) u t+\frac{\left.\left(\left(\rho^{2}(u-1)-u+\frac{1}{2}\right)-\frac{1}{2} \zeta u \frac{t}{T}\left(\rho^{2}-1\right)\right)+\rho \lambda\right)}{\xi^{\frac{1}{2}}}\right] \\
\bar{B}=\left\{\left[(u-1) \rho^{2}-u+\frac{1}{2}\right] T+u t\left(1-\rho^{2}\right)\right\} \zeta+\lambda \rho T
\end{gathered}
$$

Remark 6. The pricing of Geometric Asian options in Heston's model has been investigated by Kim and Wee in [KW11]. They express the joint moment generating function of returns and integral average in terms of some series expansions. In fact, their series can be summed in closed form in terms of hypergeometric functions and agrees with our results above. 


\subsection{The Bates model}

In a model proposed by Bates Bat96, a jump component is introduced in the previous dynamics for the log-returns by means of the compound Poisson process $Z$ :

$$
Z_{t}=\sum_{i=1}^{N_{t}} J_{i}
$$

where $N$ is a standard Poisson process with intensity $\nu>0$ and $\left(J_{i}\right), i=1,2,3, \ldots$, are independent random variables, all having a normal distribution with mean $\gamma$ and standard deviation $\delta$. In such a case the Lévy measure of $Z$ is given by:

$$
U(d x)=\frac{\nu}{\delta \sqrt{2 \pi}} \exp \left[-\frac{(x-\gamma)^{2}}{2 \delta^{2}}\right],
$$

and the cumulant function of $Z$ takes the form:

$$
\kappa(z)=\nu\left(e^{\gamma z+\delta^{2} z^{2} / 2}-1\right)
$$

The dynamics of discounted log-returns under the risk-neutral measure is then given by:

$$
d X_{t}=\left(-\kappa(1)-\frac{1}{2} V_{t}\right) d t+\sqrt{V_{t}} d W_{t}^{1}+d Z_{t},
$$

and the dynamics of the volatility is the same as that proposed by the Heston model, namely

$$
d V_{t}=\lambda\left(\theta-V_{t}\right) d t+\zeta \sqrt{V_{t}} d W_{t}^{2} .
$$

The affine characteristics are

$$
F(u, w)=\lambda \theta w+\kappa(u)-u \kappa(1), \quad R(u, w)=\frac{1}{2}\left(u^{2}-u\right)+\frac{\zeta^{2}}{2} u_{2}^{2}-\lambda w+u w \rho \zeta .
$$

If $\nu \rightarrow 0$, then we obtain the Heston stochastic volatility model Hes93. If $\zeta \rightarrow 0$ and $V_{0}=\theta$ then $V_{t}=\theta$ we obtain the Merton jump-diffusion model Mer76. Consequently we might consider the Bates model as an extension of a Merton model to the case of stochastic volatility, or as an extension of the Heston stochastic volatility model to the case of jumps in the asset prices.

In the Bates model the Riccati equations for the average price are

$$
\begin{aligned}
& \dot{\phi}=\lambda \theta \psi+\kappa(u t)-u t \kappa(1), \quad \phi(0)=0 \\
& \dot{\psi}=\frac{\zeta^{2}}{2} \psi^{2}-(\lambda-\rho \zeta u t) \psi+\frac{1}{2} u t(u t-1), \quad \psi(0)=0 .
\end{aligned}
$$

We observe that the equation for $\psi$ is exactly the same as in the Heston model and and $\phi$ equals the corresponding quantity from the Heston model plus an integral of the cumulant of the jumps (quite easy to compute):

$$
\phi(t)=\phi_{H}(t)+\int_{0}^{t} \kappa(u s) d s-u t \kappa(1),
$$

where $\phi_{H}$ is given above in (67). This is due to the fact, that the jumps are independent of the continuous part.

For the average strike we obtain the same $\psi$ as in the Heston model, while the $\phi$ is provided by the following expression (also easy to compute):

$$
\phi(t)=\phi_{H}(t)+\int_{0}^{t} \kappa\left(u\left(\frac{t}{T}-1\right) u+1\right) d s-\frac{u t}{T} \kappa(1)+(1-u) \kappa(1)
$$

where $\phi_{H}$ is given above, using (70) and (71). 


\subsection{The Turbo-Bates model}

In Bat00 Bates introduced a refinement of the previous model with state-dependet jump intensity. Following [KR11, Sec.6.2] we will consider a simplified version with only one variance factor. The risk-neutral dynamics for log-returns are given by

$$
\begin{gathered}
d X_{t}=\left(-\nu_{0} \kappa(1)-\left(\frac{1}{2}+\nu_{1} \kappa(1)\right) V_{t}\right) d t+\sqrt{V_{t}} d W_{t}^{1}+\int_{D} x \tilde{N}\left(V_{t}, d t, d x\right) \\
d V_{t}=-\lambda\left(V_{t}-\theta\right) d t+\zeta \sqrt{V_{t}} d W_{t}^{2}
\end{gathered}
$$

where $\lambda, \theta, \zeta>0$ as before and the Brownian motion $W^{1}, W^{2}$ are correlated with correlation coefficient $\rho$. The jump component is given by $\tilde{N}\left(V_{t}, d t, d x\right)=N\left(V_{t}, d t, d x\right)-\mu\left(V_{t}, d t, d x\right)$, where $N\left(V_{t}, d t, d x\right)$ is a Poisson random measure and its predictable compensator $\mu\left(V_{t}, d t, d x\right)=$ $\left(\nu_{0}+\nu_{1} V_{t}\right) F(d x) d t$, and $F$ is some fixed jump size distribution.

The affine characteristics are

$F(u, w)=\nu_{0} \kappa(u)-u \nu_{0} \kappa(1) 0+\lambda \theta w, \quad R(u, w)=\frac{1}{2}\left(u^{2}-u\right)+\frac{\zeta^{2}}{2} w^{2}-\lambda w+\rho \zeta u w+\nu_{1} \kappa(u)-u \nu_{1} \kappa(1)$

where $\kappa(u)$ is the cumulant generating function of $F$.

The Riccati equations for the average price are

$$
\begin{aligned}
& \dot{\phi}=\lambda \theta \psi+\nu_{0} \kappa(u t / T)-u t / T \nu_{0} \kappa(1), \quad \phi(0)=0 \\
& \dot{\psi}=\frac{\zeta^{2}}{2} \psi^{2}-(\lambda-\rho \zeta u t / T) \psi+\frac{1}{2} u t / T(u t / T-1)+\nu_{1} \kappa(u t / T)-u t / T \nu_{1} \kappa(1), \quad \psi(0)=0
\end{aligned}
$$

and for the average strike

$$
\begin{aligned}
\dot{\phi} & =\alpha \theta \psi+\nu_{0} \kappa(u(t / T-1)+1)-\left(\frac{u t}{T}+(1-u)\right) \nu_{0} \kappa(1), \quad \phi(0)=0 \\
\dot{\psi} & =\frac{1}{2} u^{2}(t / T-1)^{2}+u(t / T-1)+\frac{\zeta^{2}}{2} \psi^{2}-\beta \psi+\rho \zeta(u(t / T-1)+1) \psi+ \\
& +\lambda_{1} \kappa((u(t / T-1)+1))-\left(\frac{u t}{T}+(1-u)\right) \nu_{1} \kappa(1), \\
\psi(0)=0 &
\end{aligned}
$$

\subsection{Barndorff-Nielsen-Shephard model}

The BNS model has been introduced by Ole Barndorff-Nielsen and Neil Shephard. BNS01, BNNS02, NV03, HS09. The model is constructed from a subordinator, called background driving Lévy process (BDLP), with cumulant generating function

$$
\kappa(\theta)=\log E\left[e^{\theta Z(1)}\right],
$$

which exists for $\Re(\theta)<\ell$ with some real number $\ell>0$. The instantaneous variance process $(V(t), t \geq 0)$ is described by the following stochastic differential equation of Ornstein-Uhlenbeck type,

$$
d V(t)=-\lambda V(t-) d t+d Z_{\lambda}(t),
$$

with $V_{0}>0$ and $\lambda>0$ given real numbers. The logarithmic return process $(X(t), t \geq 0)$ is given by:

$$
d X(t)=\left(-\kappa(\rho)-\frac{1}{2} V(t-)\right) d t+\sqrt{V(t-)} d W(t)+\rho d Z_{\lambda}(t), \quad X(0)=0,
$$


with parameters $\mu \in, \beta \in, \rho \leq 0$. The affine characteristics are

$$
F(u, w)=\lambda k(w+\rho u)-u \lambda k(\rho), \quad R(u, w)=\frac{1}{2}\left(u^{2}-u\right)-\lambda w .
$$

Riccati equations for average price are

$$
\begin{array}{ll}
\dot{\phi}=\lambda k(\psi+\rho u t / T)-u t / T \lambda k(\rho) & \phi(0)=0 \\
\dot{\psi}=\frac{1}{2}\left(u^{2} t^{2} / T^{2}-u t / T\right)-\lambda \psi & \psi(0)=0 .
\end{array}
$$

We remark the equation for $\psi$ is linear and can be solved explicitly, giving

$$
\psi(t)=\frac{u^{2}}{2 T^{2}} f_{2}(t)-\frac{u}{t T} f_{1}(t) .
$$

with

$$
f_{0}(t)=\frac{1-e^{-\lambda t}}{\lambda}, \quad f_{1}(t)=\frac{t}{\lambda}-\frac{1-e^{-\lambda t}}{\lambda^{2}}, \quad f_{2}(t)=\frac{t^{2}}{\lambda}-\frac{2 t}{\lambda^{2}}+\frac{2\left(1-e^{-\lambda t}\right)}{\lambda^{3}} .
$$

The equation for $\phi$ yields an integral

$$
\phi(t)=\int_{0}^{t} \lambda k(\psi(s)+\rho u s / T)-\frac{t^{2}}{2} \frac{u}{T} \lambda k(\rho)
$$

Riccati equations for average strike are

$$
\begin{array}{ll}
\dot{\phi}=\lambda k(\psi+\rho(u(t / T-1)+1)) & \phi(0)=0 \\
\dot{\psi}=\frac{1}{2} u^{2}(t / T-1)^{2}+u(t / T-1)-\lambda \psi & \psi(0)=0 .
\end{array}
$$

The solution is quite analogous, now with

$$
\psi(t)=\frac{u^{2}}{2 T^{2}} f_{2}(T)+\frac{u}{T}\left(\frac{1}{2}-u\right) f_{1}(T)+\left(\frac{u^{2}}{2}-\frac{u}{2}\right) f_{0}(T) .
$$

and the integral

$$
\phi(t)=\int_{0}^{T} \lambda k\left(\psi(s)+\rho\left(\left(\frac{s}{T}-1\right) u+1\right)\right) d s-((1-T) u+T) \lambda k(\rho) .
$$

The results agred 1 with those from [HS11, which were obtained by a different technique without employing the general affine framework and Riccati equations.

\subsection{OU time-changed Lévy processes}

Time-changed Levy processes have been introduced by P. Carr, H. Geman, D. Madan and M. Yor CGMY03 in order to improve Levy models performances in describing asset price dynamics. We shall concentrate our attention on time changes based on processes satisfying a stochastic differential equation of an Ornstein-Uhlenbeck or a square-roote (CIR) type. Let $L$ be a Lévy process with cumulant function

$$
\theta(u)=\log E\left[e^{u L(1)}\right] .
$$

\footnotetext{
${ }^{1}$ Actually term $-u / 2$ is missing in [HS11, (47)] and should be included there.
} 
Then we define

$$
X_{t}=L(\Gamma(t))
$$

where $\Gamma(t)$ is a non-negative increasing process independent of $L$. Here we would like to use a very popular time change, namely an integrated Ornstein-Uhlenbeck (OU) type process.

Definition 1 (OU time-change). The OU time-change model is given as

$$
\Gamma(t)=\int_{0}^{t} V(s) d s,
$$

where $V$ is now given as solution of the $S D E$

$$
d V(t)=-\lambda V(t) d t+d U(t)
$$

with $U$ being a pure jump subordinator with cumulant function $\kappa(u)$.

The affine characteristics are

$$
F(u, w)=\lambda \kappa(w), \quad R(u, w)=-\lambda w+\theta(u) .
$$

For the Riccati equations we get from (41) and (111)

$$
\begin{array}{ll}
\dot{\phi}=\lambda \kappa(\psi) & \phi(0)=0 \\
\dot{\psi}=-\lambda \psi+\theta(u t) & \psi(0)=0 .
\end{array}
$$

The Riccati equations for average strike are

$$
\begin{array}{ll}
\dot{\phi}=\lambda \kappa(\psi)+q-r & \phi(0)=0 \\
\dot{\psi}=-\lambda \psi+\theta(u(t / T-1)+1) & \psi(0)=0
\end{array}
$$

Let us consider a concrete example of a time-changed Lévy given by a Kou double exponential Lèvy process with time change implied by an integrated OU process. By recalling that the cumulant for the double exponential has the following expression:

$$
\kappa(u)=\nu u\left[\frac{p}{\alpha_{+}-u}-\frac{1-p}{\alpha_{-}+u}\right],
$$

where $\nu$ is the intensity of the jump process, $\alpha_{-}, \alpha_{+}$describe the exponential tails, the Riccati equations for average price have the following explicit solution:

$$
\begin{aligned}
\psi(t)= & -e^{-\lambda t} \frac{\nu}{\lambda u}\left\{\left[p \operatorname{Ei}\left(1,-\frac{\lambda \alpha_{-}}{u}\right) e^{\left(\frac{\lambda \alpha_{-}}{u}\right)} \lambda \alpha_{-} \operatorname{Ei}\left(1, \frac{\lambda \alpha_{+}}{u}\right) e^{\left(\frac{\lambda \alpha_{+}}{u}\right)} \lambda \alpha_{+}+u+p \lambda \alpha_{+} \operatorname{Ei}\left(1, \frac{\lambda \alpha_{+}}{u}\right) e^{\left(\frac{\lambda \alpha_{+}}{u}\right)}\right\}\right. \\
& -e^{-\lambda t} \frac{\nu}{\lambda u}\left\{\left[p \operatorname{Ei}\left(1,-\frac{\lambda\left(\alpha_{-}+u t\right)}{u}\right) e^{\left(\frac{\lambda\left(\alpha_{-}+u t\right)}{u}\right)} \lambda \alpha_{-} \operatorname{Ei}\left(1, \frac{\lambda\left(\alpha_{+}-u t\right)}{u}\right) e^{\left(\frac{\lambda\left(\alpha_{+}-u t\right)}{u}\right)} \lambda \alpha_{+}\right.\right. \\
& \left.+u+p \lambda \alpha_{+} \operatorname{Ei}\left(1, \frac{\lambda\left(\alpha_{+}-u t\right)}{u}\right) e^{\left(\frac{\lambda\left(\alpha_{+}-u t\right)}{u}\right)}\right\}
\end{aligned}
$$

While the average strike Riccati equations have the following solution:

$$
\psi(t)=e^{-\lambda t} \frac{\nu}{u}\left[p \lambda \alpha_{-} T \operatorname{Ei}\left(1,-\frac{\lambda \alpha_{-} T-\lambda u T-\lambda u(T-t)}{u}\right) e^{\left(\frac{\lambda \alpha_{-} T-\lambda u T-\lambda u(T-t)}{u}\right)}+u\right]
$$




$$
\begin{gathered}
-e^{-\lambda t} \frac{\nu}{u}\left[p \lambda \alpha_{+} T \operatorname{Ei}\left(1, \frac{\lambda \alpha_{+} T-\lambda u T-\lambda u(T-t)}{u}\right) e^{\left(\frac{\lambda \alpha_{+} T-\lambda u T-\lambda u(T-t)}{u}\right)}\right]+ \\
-e^{-\lambda t} \frac{\nu}{u}\left[-p \lambda \alpha_{+} T \operatorname{Ei}\left(1,-\frac{\lambda\left(\alpha_{-} T-\lambda u T-\lambda u(T-t)\right)}{u}\right) e^{\left(\frac{\lambda\left(\alpha_{-} T-\lambda u T-\lambda u(T-t)\right)}{u}\right)}\right]
\end{gathered}
$$

\subsection{CIR time-changed Lévy processes}

Another time change which has been proposed in CGMY03, for a Levy process in order to improve its performances in describing logreturns statistical behavior, is that driven by an integrated CIR process, i.e., a process satisfying the following SDE:

$$
d V_{t}=-\lambda\left(V_{t}-\theta\right) d t+\eta \sqrt{V_{t}} d W_{t} .
$$

The time-change and the returns process will be given by (109) and (108) as above.

The affine characteristics are

$$
F(u, w)=\lambda \theta w, \quad R(u, w)=\frac{\eta^{2}}{2} w^{2}-\lambda w+\kappa(u) .
$$

where $\kappa(u)$ is the cumulant generating function of the Lévy process.

From (41) and (122) we obtain the Riccati equations for average price

$$
\begin{array}{ll}
\dot{\phi}=\lambda \theta \psi & \phi(0)=0 \\
\dot{\psi}=\frac{\eta^{2}}{2} \psi^{2}+-\lambda \psi+\kappa(u t) & \psi(0)=0 .
\end{array}
$$

For average strike

$$
\begin{array}{ll}
\dot{\phi}=\lambda \theta \psi+q-r & \phi(0)=0 \\
\dot{\psi}=\frac{\eta^{2}}{2} \psi^{2}+-\lambda \psi+\kappa(u(t / T-1)+1) & \psi(0)=0
\end{array}
$$

Let us consider a concrete example of a time-changed Lévy given by a Kou double exponential Lèvy process with time change implied by an integrated CIR process. By recalling that the cumulant for the double exponential has the following expression:

$$
\kappa(u)=\nu u\left[\frac{p}{\alpha_{+}-u}-\frac{1-p}{\alpha_{-}+u}\right],
$$

where $\nu$ is the intensity of the jump process, $\alpha_{-}, \alpha_{+}$describe the exponential tails, the Riccati equation for $\psi$ becomes:

$$
\dot{\psi}=\frac{\eta^{2}}{2}-\nu \dot{\psi} \psi^{2}+\lambda u\left[\frac{p}{\nu_{-}-u}-\frac{1-p}{\nu_{+}+u}\right], \psi_{w}(0)=0 .
$$

For a symmetric jump distribution, i.e., $p=1 / 2$ and $\alpha_{+}=\alpha_{-}$we can provide an explicit solution in terms of Heun's confluent hypergeometric function $C$, see [Ron95, SK10]:

$$
\begin{gathered}
\psi(t)=-\frac{2}{\eta^{2}} \frac{y_{2}^{\prime}(0) y_{1}^{\prime}(t)-y_{1}^{\prime}(0) y_{2}^{\prime}(t)}{y_{2}^{\prime}(0) y_{1}(t)-y_{1}^{\prime}(0) y_{2}(t)} . \\
y_{1}=\exp \left(-\frac{\lambda t}{2}\right)\left(\alpha_{+}^{2}-u^{2} t^{2}\right) C\left(0,-\frac{1}{2}, 1, \frac{-\lambda^{2} \alpha_{+}^{2}+2 e t^{2} \nu \alpha_{+}}{16 u^{2}}, \frac{8 u^{2}+\lambda^{2} \alpha_{+}^{2}}{16 u^{2}}, \frac{u^{2} t^{2}}{\alpha_{+}^{2}}\right) \\
y_{2}=\exp \left(-\frac{\lambda t}{2}\right)\left(\alpha_{+}^{2}-u^{2} t^{2}\right) C\left(0,+\frac{1}{2}, 1, \frac{-\lambda^{2} \alpha_{+}^{2}+2 e t^{2} \nu \alpha_{+}}{16 u^{2}}, \frac{8 u^{2}+\lambda^{2} \alpha_{+}^{2}}{16 u^{2}}, \frac{u^{2} t^{2}}{\alpha_{+}^{2}}\right)
\end{gathered}
$$




\section{Concluding Remarks}

In this paper we have just provided a framework for Geometric Asian options valuation and we have shown that this valuation problem, through the general affine process approach, can be reduced to solving some generalized Riccati equations and that in many relevant cases these equations admit close-form solutions. The final step of the present valuation procedure requires the numerical inversion of a Laplace transform. This computation, which has become quite standard in option pricing, nevertheless requires some care especially when complicated special functions, like those considered insofar, are involved. The research of a fast and accurate algorithm providing such inversion will be the subject of our future investigation together with an extensive comparison of the numerical methods available for Geometric Asian option pricing in affine stochastic volatility models. As we mentioned in Section 5] proper integrability conditions must be verified in order to apply our general pricing results: the existence of all the involved cumulant functions must be assured; this can be investigated through the analysis of the singularities of the special functions introduced. This subject, together with a systematic numerical illustration of the present results will be the subject of our future investigation and will be collected in a separate paper.

\section{A Laplace formulae}

Lemma 3. Suppose we are given real numbers $S_{0}>0, K>0$, and $a>1,0<b<1, c<0$. Then we have for all $x \in \mathbb{R}$ the formulas

$$
\begin{aligned}
& \left(e^{x}-K\right)_{+}=\frac{1}{2 \pi i} \int_{a-i \infty}^{a+i \infty}\left(\frac{1}{K}\right)^{u} \frac{K}{u(u-1)} e^{u x} d u, \\
& \left(K-e^{x}\right)_{+}=\frac{1}{2 \pi i} \int_{c-i \infty}^{c+i \infty}\left(\frac{1}{K}\right)^{u} \frac{K}{u(u-1)} e^{u x} d u,
\end{aligned}
$$

and

$$
\left(e^{x}-K\right)_{+}-e^{x}=\frac{1}{2 \pi i} \int_{b-i \infty}^{b+i \infty}\left(\frac{1}{K}\right)^{u} \frac{K}{u(u-1)} e^{u x} d u .
$$

Proof. Let $f(x)=\left(e^{x}-K\right)_{+}$. An elementary calculation provides the (bilateral) Laplace transform of $f$, namely

$$
\int_{-\infty}^{+\infty} f(x) e^{-u x} d x=\left(\frac{1}{K}\right)^{u} \frac{K}{u(u-1)}
$$

for $\Re u>1$. Now $f$ is continuous and has locally bounded variation, which are sufficient conditions to guarantee that the Laplace inversion integral (with Bromwhich contour) yields the original function, that is (132). See [Doe71, Satz 4.4.1, P.210]. The proof for (133) and (134) is similar. 


\section{References}

[AG03] Hansjörg Albrecher and Marc Goovaerts. Static hedging of Asian options under Lévy models: The comonotonicity approach. Preprint, Graz University of Technology, 2003.

[Alb04] Hansjörg Albrecher. The valuation of Asian options for market models of exponential Lévy type. Preprint, 2004. check!

[AP04] Hansjörg Albrecher and Martin Predota. On Asian option pricing for NIG Lévy processes. Journal of Computational and Applied Mathematics, 172(1):153-168, 2004.

[Bat96] D. Bates. Jumps and stochastic volatility: the rexchange rate processes implicit in deutsche mark options. The Review of Financial Studies, 9:69-107, 1996. check!

[Bat00] D. Bates. Post-'87 crash fears in the s\&p 500 futures option market. Journal of Econometrics, 94(1-2):181-238, 2000. check!

[BGK07] Fred Espen Benth, Martin Groth, and Rodwell Kufakunesu. Valuing volatility and variance swaps for a non-Gaussian Ornstein-Uhlenbeck stochastic volatility model. Applied Mathematical Finance, 14(4):347-363, 2007.

[BNNS02] Ole E. Barndorff-Nielsen, Elisa Nicolato, and Neil Shephard. Some recent developments in stochastic volatility modelling. Quantitative Finance, 2(1):11-23, 2002. Special issue on volatility modelling.

[BNS01] Ole E. Barndorff-Nielsen and Neil Shephard. Non-Gaussian Ornstein-Uhlenbeckbased models and some of their uses in financial economics. Journal of the Royal Statistical Society. Series B. Statistical Methodology, 63(2):167-241, 2001.

[CGMY03] Peter Carr, Hélyette Geman, Dilip B. Madan, and Marc Yor. Stochastic volatility for Lévy processes. Mathematical Finance, 13(3):345-382, 2003.

[CLW12] Peter Carr, Roger Lee, and Liuren Wu. Variance swaps on time-changed Lévy processes. Finance and Stochastics, 16(2):335-355, 2012.

[CW04a] Peter Carr and Liuren Wu. Time-changed Lévy processes and option pricing. Journal of Financial Economics, 71(1):113-141, 2004.

[CW04b] Ying Lok Cheung and Hoi Ying Wong. Geometric Asian options: valuation and calibration with stochastic volatility. Quantitative Finance, 4(3):301-314, 2004.

[DFS03] D. Duffie, D. Filipović, and W. Schachermayer. Affine processes and applications in finance. The Annals of Applied Probability, 13(3):984-1053, 2003.

[Doe71] Gustav Doetsch. Handbuch der Laplace-Transformation. Band I: Theorie der Laplace-Transformation. Birkhäuser Verlag, Basel, 1971.

[Duf01] Daniel Dufresne. The integral of geometric Brownian motion. Advances in Applied Probability, 33(1):223-241, 2001.

[Duf05] Daniel Dufresne. Bessel processes and Asian options. In Michèle Breton and Hatem Ben-Ameur, editors, Numerical methods in finance, volume 9 of GERAD 25th Anniversary Series, pages 35-57. Springer, New York, 2005. 
[EPS08] Ernst Eberlein, Antonis Papapantoleon, and Albert N. Shiryaev. On the duality principle in option pricing: semimartingale setting. Finance and Stochastics, 12(2):265292, 2008.

[Fel51] William Feller. Two singular diffusion problems. Annals of Mathematics, 54:173-182, 1951.

[FH03] Jean-Pierre Fouque and Chuan-Hsiang Han. Pricing Asian options with stochastic volatility. Quantitative Finance, 3(5):353-362, 2003.

[FM08] Gianluca Fusai and Attilio Meucci. Pricing discretely monitored Asian options under Lévy processes. Journal of Banking and Finance, 32(10):2076-2088, 2008.

[Gla04] Paul Glasserman. Monte Carlo Methods in Financial Engineering. Springer, 2004.

[GM11] Emmanuel Gobet and Mohammed Miri. Weak approximation of averaged diffusion processes. Preprint, 2011.

[GY93] Hélyette Geman and Marc Yor. Bessel processes, Asian options, and perpetuities. Mathematical Finance, 3(4):349-375, 1993.

[Hes93] Steven L. Heston. A closed-form solution for options with stochastic volatility with applications to bond and currency options. Rev. Fin. Studies, 6:327-343, 1993.

[HKK06] Friedrich Hubalek, Jan Kallsen, and Leszek Krawczyk. Variance-optimal hedging for processes with stationary independent increments. The Annals of Applied Probability, 16(2):853-885, 2006.

[HS09] Friedrich Hubalek and Carlo Sgarra. On the Esscher transforms and other equivalent martingale measures for Barndorff-Nielsen and Shephard stochastic volatility models with jumps. Stochastic Processes and their Applications, 119(7):2137-2157, 2009.

[HS11] Friedrich Hubalek and Carlo Sgarra. On the explicit evaluation of the geometric Asian options in stochastic volatility models with jumps. Journal of Computational and Applied Mathematics, 235(11):3355-3365, 2011.

[Kal06] Jan Kallsen. A didactic note on affine stochastic volatility models. In From stochastic calculus to mathematical finance, pages 343-368. Springer, Berlin, 2006.

[Kat01] Harry Kat. Structured Equity Derivatives: The Definite Guide to Exotic Options and Structured Notes. John Wiley, 2001.

[KMKV11] Jan Kallsen, Johannes Muhle-Karbe, and Moritz Voß. Pricing options on variance in affine stochastic volatility models. Mathematical Finance, 21(4):627-641, 2011.

[KR08] Martin Keller-Ressel. Affine processes - Theory and applications in finance. Dissertation, Vienna University of Technology, 2008.

[KR11] Martin Keller-Ressel. Moment explosions and long-term behavior of affine stochastic volatility models. Mathematical Finance, 21(1):73-98, 2011.

[KRST10] Martin Keller-Ressel, Walter Schachermayer, and Joseph Teichmann. Affine processes are regular. Preprint, 2010.

[KW11] Bara Kim and In-Suk Wee. Pricing of geometric Asian options under Heston's stochastic volatility model. Quantitative Finance, iFirst:1-15, 2011. 
[Mer76] R. Merton. Option pricing when underlying stock returns are discontinuous. The Journal of Financial Economics, 3:125-144, 1976. check!

[MP98] M.A. Milevsky and S.E. Posner. Asian options, the sum of lognormals and the reciprocal gamma distribution. Journal of Financial and Quantitative Analysis, 33(3):409-422, 1998.

[NV03] Elisa Nicolato and Emmanouil Venardos. Option pricing in stochastic volatility models of the Ornstein-Uhlenbeck type. Mathematical Finance, 13(4):445-466, 2003.

[Pap07] Antonis Papapantoleon. Applications of semimartingales and Lévy processes in finance: duality and valuation. Phd thesis, University of Freiburg, 2007.

[Pen06] Bin Peng. Pricing geometric Asian options under the CEV process. International Economic Journal, 20(4):515-522, 2006.

[PZ03] Andrei D. Polyanin and Valentin F. Zaitsev. Handbook of exact solutions for ordinary differential equations. Chapman \& Hall/CRC, Boca Raton, FL, second edition, 2003.

[Rei72] William T. Reid. Riccati differential equations. Academic Press, New York, 1972. Mathematics in Science and Engineering, Vol. 86.

[Ron95] A. Ronveaux, editor. Heun's differential equations. Oxford Science Publications. The Clarendon Press Oxford University Press, New York, 1995.

[Sch08] Michael Schröder. On constructive complex analysis in finance: explicit formulas for Asian options. Quarterly of Applied Mathematics, 66(4):633-658, 2008.

[Sep08] Artur Sepp. Pricing options on realized variance in the Heston model with jumps in returns and volatility. Journal of Computational Finance, 11(4):33-70, 2008.

[SGD00] S. Simon, M.J. Goovaerts, and J. Dhaene. An easily computable upper bound for the price of an arithmetic Asian option. Insurance: Mathematics and Economics, 26(2-3):175-183, 2000.

[SK10] B. D. Sleeman and V. B. Kuznetsov. Heun functions. In NIST handbook of mathematical functions, pages 709-721. U.S. Dept. Commerce, Washington, DC, 2010.

[Sla60] L. J. Slater. Confluent hypergeometric functions. Cambridge University Press, New York, 1960.

[VDL $\left.{ }^{+} 06\right]$ M. Vanmaele, G. Deelstra, J. Liinev, J. Dhaene, and M. J. Goovaerts. Bounds for the price of discrete arithmetic Asian options. Journal of Computational and Applied Mathematics, 185(1):51-90, 2006.

[VX04] Jan Večeř and Mingxin Xu. Pricing Asian options in a semimartingale model. Quantitative Finance, 4(2):170-175, 2004.

[WHD95] Paul Wilmott, Sam Howison, and Jeff Dewynne. The mathematics of financial derivatives. Cambridge University Press, Cambridge, 1995.

[ZO13] B. Zhang and C. W. Oosterlee. Efficient pricing of European-style Asian options under exponential Lévy processes based on Fourier cosine expansions. SIAM Journal on Financial Mathematics, 4(1):399-426, 2013. 